\title{
Weyl semimetal/insulator transition from holography
}

\section{Yan Liu and Junkun Zhao}

Department of Space Science, and International Research Institute of Multidisciplinary Science, Beihang University, Beijing 100191, China

E-mail: yanliu@buaa.edu.cn, junkunzhao@buaa.edu.cn

ABSTRACT: We study a holographic model which exhibits a quantum phase transition from the strongly interacting Weyl semimetal phase to an insulating phase. In the holographic insulating phase there is a hard gap in the real part of frequency dependent diagonal conductivities. However, the anomalous Hall conductivity is nonzero at zero frequency, indicting that it is a Chern insulator. This holographic quantum phase transition is always of first order, signified by a discontinuous anomalous Hall conductivity at the phase transition, in contrast to the very continuous holographic Weyl semimetal/trivial semimetal phase transition. Our work reveals the novel phase structure of strongly interacting Weyl semimetal.

KEYWORDS: Holography and condensed matter physics (AdS/CMT), Gauge-gravity correspondence

ARXiv EPrint: 1809.08601 


\section{Contents}

1 Introduction 1

2 Holographic setup $\quad 3$

2.1 Zero temperature solutions 5

$\begin{array}{lll}2.2 & \text { Asymptotic expansions and free energy } & 7\end{array}$

3 Transport properties of the dual theory $\quad 9$

3.1 Longitudinal conductivities 9

$\begin{array}{ll}3.2 & \text { Transverse conductivities } \\ 3.3 & \text { Phase }\end{array}$

$\begin{array}{lll}3.3 & \text { Phase diagram } & 12\end{array}$

4 Conclusion and discussion $\quad 14$

$\begin{array}{ll}\text { A Weakly coupled field theory for Weyl semimetal } & 16\end{array}$

$\begin{array}{ll}\text { B Equations of motion at finite temperature } & 17\end{array}$

$\begin{array}{lll}\text { B.1 Near horizon } & 18\end{array}$

$\begin{array}{lll}\text { B.2 } & \text { Asymptotic behavior and free energy } & 18\end{array}$

$\begin{array}{ll}\text { C Schrodinger potential approach to conductivities } & 19\end{array}$

\section{Introduction}

Weyl semimetal is a nontrivial topological gapless state and exhibits lots of exotic novel and robust properties, including chiral anomaly and Fermi arc etc. It has been a research focus recently as they are both experimentally important and theoretically interesting [13]. On the one hand, they are ideal systems to test the macroscopic effects due to quantum anomaly [4], including chiral magnetic effect and transport effects induced by the mixed axial-gravitational anomaly. On the other hand, Weyl semimetal is a novel kind of topological quantum matter which goes beyond the Landau-Ginzburg's paradigm for classification of states of matter. Similar to graphene systems [5], Weyl semimetal systems could be strongly coupled and do not possess well-defined quasiparticles. It is theoretically challenging and important to study strongly interacting Weyl semimetals, to go beyond the conventional approach on topological states of matter based on topological band theory or weakly coupled theory.

The holographic correspondence maps the difficult question of strongly interacting field theory to a tractable weakly coupled gravitational problem. There have been lots of remarkable applications of holography to tackle the strongly interacting condensed matter questions [6-8]. In particular, holographic models for strongly interacting Weyl semimetals 
have been constructed in $[9,10]$ in which the anomalous Hall conductivity is an order parameter to characterize the quantum topological phase transition. ${ }^{1}$ The effects of the surface state [13] and topological invariants [14] in this holographic model exhibit key features of topological Weyl semimetals. Therefore with strong interaction topological Weyl semimetal still exits and holography is a practical tool to explore its property. Moreover, the nontrivial topological structure in the strongly interacting system can be revealed from the gravitational bulk physics $[14,15]$. There exist two bulk matter fields in which one field is to generate a gap in the dual theory while the other matter field is to deform the Fermi points to a topologically nontrivial configuration (Weyl points or nodal lines). The different topological phases arise due to the different IR solutions in the bulk which are adiabatically disconnected and only one of the matters fields dominates in each solution. From the holographic model a nontrivial prediction is that the presence of odd viscosity is due to mixed axial-gravitational anomaly [16]. Other various interesting aspects of holographic Weyl semimetals have been explored, including optical conductivity [17], axial Hall conductivity [18], disorder effect on topological phase transition [19] and the butterfly velocity [20].

In condensed matter systems, from weakly coupled theory Weyl semimetal can go through a quantum phase transition to a normal band insulator [21, 22] or to Chern insulator $[21,23,24]$ etc. $^{2}$ It would be extremely interesting to explore the phase diagram of strongly interacting topological Weyl semimetal from holography. In the previous holographic models $[9,10]$ only a portion of degrees of freedom are gapped in the trivial phase and Weyl semimetal phase goes to a trivial semimetal phase after the phase transition. This paper aims to provide a holographic model to describe a quantum phase transition from Weyl semimetal to a phase in which all the degrees of freedom are gapped, namely, the trivial phase is instead an insulating phase. In doing so we start from the most generic holographic Weyl semimetal model by using the Stueckelberg trick to replace the complex scalar field in [9] by two real scalar fields and introduce the most general dilatonic coupling. Writing the equations for fluctuations of gauge fields into a Schrodinger equation, we can get the condition for the dilatonic couplings to produce the insulating phase. With a proper choice of dilatonic coupling and potential terms, we could realise a holographic topological quantum phase transition between strongly interacting Weyl semimetal phase and Chern insulator (3+1D anomalous Hall state) phase. Then we show the evidences of the phase transition from the perspectives of free energy and conductivities.

Our paper is organized as follows. In section 2, we introduce a generalized holographic model with dilatonic coupling to realise the quantum phase transition from Weyl semimetal phase to insulator phase and show that it is a first order phase transition. In section 3 , the conductivities of the dual theory are explored by studying the vector gauge field fluctuations above the background geometry. Evidence for the insulating phase being a Chern insulator is discussed. In section 4, we conclude and discuss the open problems. Appendices A, B, C are devoted to the details of the field theory model, the finite temperature equations of motion for holographic model and the Schrodinger potential approach for conductivities.

\footnotetext{
${ }^{1}$ There are also other holographic models for Weyl semimetal, e.g. from the perspectives of fermionic spectral function [11] and top-down models [12].

${ }^{2}$ See appendix A for examples from field theoretical approach.
} 


\section{Holographic setup}

We shall start from the most general holographic system which duals to an anomalous system with $\mathrm{U}(1)_{V} \times \mathrm{U}(1)_{A}$. This $\mathrm{U}(1)_{A}$ will be explicitly broken by turning on a source term which plays the similar role of mass effect in the dual field theory. With the dilatonic coupling, the generic holographic model is

$$
\begin{aligned}
\mathcal{S}= & \int d^{5} x \sqrt{-g}\left[\frac{1}{2 \kappa^{2}}\left(R+12-\frac{1}{2}(\partial \phi)^{2}-V(\phi)\right)-\frac{Y(\phi)}{4 e^{2}} \mathcal{F}^{2}-\frac{Z(\phi)}{4 e_{2}^{2}} F^{2}\right. \\
& \left.+\frac{\alpha}{3} \epsilon^{a b c d e} A_{a}\left(F_{b c} F_{d e}+3 \mathcal{F}_{b c} \mathcal{F}_{d e}\right)-\frac{W(\phi)}{2}\left(A_{a}-\partial_{a} \theta\right)^{2}\right]+S_{\mathrm{GH}}+S_{\text {c.t. }}
\end{aligned}
$$

with the vector gauge field strength $\mathcal{F}_{a b}=\partial_{a} V_{b}-\partial_{b} V_{a}$ and axial gauge field strength $F_{a b}=\partial_{a} A_{b}-\partial_{b} A_{a}$. Here $V_{a}$ and $A_{a}$ correspond to vector and axial current respectively. $\kappa$ is the gravitational constant, $e, e_{2}$ are bulk vector and axial gauge coupling constants. Note that the scalar fields $\phi$ and $\theta$ are real. The action is invariant under the gauge transformation $\theta \rightarrow \theta+\chi, A_{a} \rightarrow A_{a}+\partial_{a} \chi$. One can recover the holographic Weyl semimetal model in [9] via $\Phi=\frac{1}{\sqrt{2}} \phi e^{i \theta}$ which is axially charged under the axial symmetry, and choose $Y(\phi)=Z(\phi)=1, W(\phi)=q^{2} \phi^{2}, V(\phi)=\frac{m^{2}}{2} \phi^{2} . S_{\mathrm{GH}}$ is the Gibbons-Hawking boundary action and $S_{\text {c.t. }}$ is the counterterm that renormalized the on-shell action. Their explicit forms are shown in (B.19) and (B.20) of appendix B. The model (2.1) is a generic holographic model for Weyl semimetal. ${ }^{3}$

From now on we set $2 \kappa^{2}=e^{2}=e_{2}^{2}=1 .{ }^{4}$ The equations of motion of the system are

$$
\begin{aligned}
R_{a b}-\frac{1}{2} g_{a b}(R+12)-\frac{1}{2} T_{a b} & =0, \\
\nabla_{b}\left(Y(\phi) \mathcal{F}^{b a}\right)+2 \alpha \epsilon^{a b c d e} F_{b c} \mathcal{F}_{d e} & =0, \\
\nabla_{b}\left(Z(\phi) F^{b a}\right)+\alpha \epsilon^{a b c d e}\left(F_{b c} F_{d e}+\mathcal{F}_{b c} \mathcal{F}_{d e}\right)-W(\phi)\left(A^{a}-\nabla^{a} \theta\right) & =0, \\
\nabla_{a} \nabla^{a} \phi-\frac{\partial_{\phi} Y(\phi)}{4} \mathcal{F}^{2}-\frac{\partial_{\phi} Z(\phi)}{4} F^{2}-\frac{\partial_{\phi} W(\phi)}{2}\left(A_{a}-\partial_{a} \theta\right)^{2}-\partial_{\phi} V(\phi) & =0, \\
\nabla_{a}\left[W(\phi)\left(A^{a}-\nabla^{a} \theta\right)\right] & =0,
\end{aligned}
$$

where

$$
\begin{aligned}
T_{a b}= & Y(\phi)\left[\mathcal{F}_{a c} \mathcal{F}_{b}{ }^{c}-\frac{1}{4} g_{a b} \mathcal{F}^{2}\right]+Z(\phi)\left[F_{a c} F_{b}^{c}-\frac{1}{4} g_{a b} F^{2}\right] \\
& +W(\phi)\left[\left(A_{a}-\partial_{a} \theta\right)\left(A_{b}-\partial_{b} \theta\right)-\frac{1}{2} g_{a b}\left(A_{c}-\partial_{c} \theta\right)^{2}\right] \\
& +\nabla_{a} \phi \nabla_{b} \phi-\frac{1}{2} g_{a b}(\partial \phi)^{2}-g_{a b} V(\phi) .
\end{aligned}
$$

\footnotetext{
${ }^{3}$ Note that $\epsilon_{a b c d e}=\sqrt{-g} \varepsilon_{a b c d e}$ with $\varepsilon_{0123 r}=1$. Similar generalisation has been made in [17] to study the optical conductivity in the quantum critical regime.

${ }^{4}$ Note that we have already set the AdS radius $L=1$. One could study the system in details without fixing $2 \kappa^{2}=e^{2}=e_{2}^{2}=1$ while introducing two additional dimensionless free parameters $\beta_{1}=\frac{e}{\sqrt{2} \kappa}$ and $\beta_{2}=\frac{e_{2}}{\sqrt{2} \kappa}$ charactering the ratios between the electromagnetic interactions and gravitational interaction following e.g. [25]. This can be achieved by rescale: $V_{a} \rightarrow \beta_{1} V_{a}, A_{a} \rightarrow \beta_{2} A_{a}, \theta \rightarrow \beta_{2} \theta$.
} 
The dual consistent currents can be obtained through the variation of the on-shell action with respect to the gauge fields,

$$
\begin{aligned}
& J^{\mu}=\lim _{r_{c} \rightarrow \infty}\left[\sqrt{-g} Y \mathcal{F}^{\mu r}+4 \alpha \sqrt{-g} \varepsilon^{r \mu \nu \rho \lambda} A_{\nu} \mathcal{F}_{\rho \lambda}+\frac{\delta S_{\text {c.t. }}}{\delta v_{\mu}}\right], \\
& J_{5}^{\mu}=\lim _{r_{c} \rightarrow \infty}\left[\sqrt{-g} Z F^{\mu r}+\frac{4}{3} \alpha \sqrt{-g} \varepsilon^{r \mu \nu \rho \lambda} A_{\nu} F_{\rho \lambda}+\frac{\delta S_{\text {c.t. }}}{\delta a_{\mu}}\right],
\end{aligned}
$$

with

$$
\begin{aligned}
& \nabla_{\mu} J^{\mu}=0 \\
& \nabla_{\mu} J_{5}^{\mu}=\lim _{r_{c} \rightarrow \infty}\left[\sqrt{-g} W\left(A^{r}-\nabla^{r} \theta\right)-\frac{\alpha}{3} \sqrt{-g} \varepsilon^{r \mu \nu \rho \lambda}\left(F_{\mu \nu} F_{\rho \lambda}+3 \mathcal{F}_{\mu \nu} \mathcal{F}_{\rho \lambda}\right)\right]+\text { c.t. }
\end{aligned}
$$

For simplicity the countertem part is not shown here. Note that the above equations are the dual Ward identities at the operator level. One can always choose the radial gauge $A_{r}=0$. For a particular state of the dual field theory, i.e. the fluctuation state around the background in the bulk, the term $-\sqrt{-g} W g^{r r} \partial_{r} \theta$ plays the role of the explicit breaking term as in the weakly coupled theory which can be found in appendix A. Since the Ward identity of conserved currents should not depend on the coupling constant of the system, it is expected that this holographic model describes a strongly interacting Weyl semimetal model.

We shall focus on the zero temperature physics. The ansatz for the background fields at zero temperature is

$$
d s^{2}=u\left(-d t^{2}+d x^{2}+d y^{2}\right)+\frac{d r^{2}}{u}+h d z^{2}, \quad A=A_{z} d z, \quad \phi=\phi(r),
$$

where fields $u, h, A_{z}, \phi$ are functions of the radial coordinate $r$ and the $\mathrm{AdS}_{5}$ boundary is located at $r \rightarrow \infty$. Note that according to the equation of motion for $\theta$, a constant solution of $\theta$ will be found and we have set it to be zero. The corresponding equations of motion are

$$
\begin{aligned}
\frac{3 u^{\prime \prime}}{u}+\phi^{\prime 2}-\frac{3 h^{\prime} u^{\prime}}{2 h u}-\frac{W A_{z}^{2}}{h u} & =0, \\
\frac{1}{4} \phi^{\prime 2}+\frac{6}{u}-\frac{3 u^{\prime}}{4 u}\left(\frac{u^{\prime}}{u}+\frac{h^{\prime}}{h}\right)-\frac{V}{2 u}-\frac{W A_{z}^{2}}{4 u h}+\frac{Z A_{z}^{\prime 2}}{4 h} & =0, \\
A_{z}^{\prime \prime}+A_{z}^{\prime}\left(\frac{2 u^{\prime}}{u}-\frac{h^{\prime}}{2 h}+\frac{\phi^{\prime} \partial_{\phi} Z}{Z}\right)-\frac{A_{z} W}{u Z} & =0, \\
\phi^{\prime \prime}+\phi^{\prime}\left(\frac{2 u^{\prime}}{u}+\frac{h^{\prime}}{2 h}\right)-\frac{\partial_{\phi} V}{u}-\frac{A_{z}^{2} \partial_{\phi} W}{2 h u}-\frac{A_{z}^{\prime 2} \partial_{\phi} Z}{2 h} & =0,
\end{aligned}
$$

where the prime is the derivative with respect to the radial coordinate $r$. We have four independent ODEs for four unknown fields.

In this paper we will choose

$$
Z(\phi)=1, \quad W(\phi)=-q_{0}\left[1-\cosh \left[\sqrt{\frac{2}{3}} \phi\right]\right], \quad V(\phi)=\frac{9}{2}\left[1-\cosh \left[\sqrt{\frac{2}{3}} \phi\right]\right] .
$$


Note that the system is invariant under the transformation $\phi \rightarrow-\phi$. When $\phi \rightarrow 0$, we have $W(\phi) \simeq \frac{q_{0}}{3} \phi^{2}$ and $V(\phi) \simeq-\frac{3}{2} \phi^{2}$. It is obvious that $q_{0}$ plays a similar role as axial charge and we restrict to $q_{0}>0$. Close to the boundary (i.e. $r \rightarrow \infty$ ), $\phi \rightarrow 0$, the potential in (2.11) has the form of $V(\phi)=\frac{1}{2} m^{2} \phi^{2}+\ldots$ with $m^{2}=-3$. The coupling $Y$ does not play any role in the background solution while it plays an important role for computing the conductivities. We set

$$
Y(\phi)=\cosh \left[\sqrt{\frac{2}{3}} \phi\right]
$$

Close to the UV boundary we have

$$
\phi=\frac{M}{r}+\ldots, \quad A_{z}=b+\ldots
$$

and a detailed expansion will be shown in subsection 2.2. $M$ and $b$ play the same roles as the sources of the scalar operator $\bar{\psi} \psi$ and chiral current $\bar{\psi} \gamma^{5} \gamma^{z} \psi$. Turning on these two sources, the dual field theory has the same structure as the weakly coupled field theory described in appendix A. In the following we shall study the bulk geometry and its free energy by tunning the parameter $M / b$ in the UV.

\subsection{Zero temperature solutions}

To study the groundstate of the system, we focus on the zero temperature solutions. ${ }^{5} \mathrm{We}$ will first find the near horizon solutions and then turn on irrelevant perturbations to generate the full solutions. At zero temperature, we find three different kinds of IR solutions.

The insulating phase. For the insulating phase, the near horizon solution is ${ }^{6}$

$$
\begin{aligned}
u & =r(1+r), \\
h & =r(1+r), \\
A_{z} & =a_{1} r^{\frac{1}{4}\left(\sqrt{1+8 q_{0}}-1\right)}, \\
\phi & =-\sqrt{\frac{3}{2}} \log \frac{r}{1+r},
\end{aligned}
$$

where $a_{1}$ is a free parameter. Note that $a_{1}$-term is the subleading term and it sources higher oder terms in $\phi$, thus different $a_{1}$ will flow the geometry to different $M / b$. The leading order of metric fields takes the form of $d s^{2}=r\left(-d t^{2}+d x^{2}+d y^{2}+d z^{2}\right)+\frac{d r^{2}}{r}$. This particular metric is known as the GPPZ gapped geometry [29] and the properties of entanglement entropy and behavior of dual scalar operators have been studied in e.g. [30, 31]. The difference is that a nontrivial $A_{z}$ will generate an anisotropic geometry. As we will show

\footnotetext{
${ }^{5}$ At finite temperature the ansatz of the background fields, the corresponding equations of motion and asymptotic expansions can be found in appendix B.

${ }^{6}$ The Ricci scalar for the near horizon geometry at the leading order is $-3 / r$ and therefore the geometry is singular near the horizon. Nevertheless the scalar potential in this solution is bounded above and satisfy the the Gubser criterion [27, 28]. Thus the singularity is acceptable and the dual field theory is not pathological.
} 
in subsection 3.1 there is a hard gap in the real part of diagonal optical conductivities while the anomalous Hall conductivity is nonzero at zero frequency, therefore this phase corresponds to a Chern insulator phase.

The Weyl semimetal phase. The near horizon solution is

$$
\begin{aligned}
u & =r^{2} \\
h & =r^{2} \\
A_{z} & =a_{0}+\frac{\phi_{0}^{2}}{4 a_{0} r} e^{-\frac{2 a_{0} \sqrt{q_{0}}}{\sqrt{3} r}} \\
\phi & =\frac{\phi_{0}}{r^{3 / 2}} e^{-\frac{a_{0} \sqrt{q_{0}}}{\sqrt{3} r}}
\end{aligned}
$$

The leading order of the IR geometry is an $\mathrm{AdS}_{5}$ geometry with a constant $A_{z}$ and we can always rescale $a_{0}$ to 1 . The exponential terms are the irrelevant perturbations. With the free parameter $\phi_{0}$, this IR geometry could flow to the whole spacetime asymptotic to $\mathrm{AdS}_{5}$ with different $M / b$. This kind of near horizon also shows up in the groundstate of the holographic superconductor [32, 33] and the holographic Weyl semimetal phase studied in [9].

The critical point. The near horizon solution is

$$
\begin{aligned}
u & =u_{0} r^{2}\left(1+\delta u r^{\alpha_{c}}\right), \\
h & =\frac{q_{0}}{9} r^{2 \beta}\left(1+\delta h r^{\alpha_{c}}\right), \\
A_{z} & =r^{\beta}\left(1+\delta a r^{\alpha_{c}}\right), \\
\phi & =\sqrt{\frac{3}{2}}\left(\log \phi_{1}\right)\left(1+\delta \phi r^{\alpha_{c}}\right) .
\end{aligned}
$$

In the case of $q_{0}=15$, we have $\left(u_{0}, \beta, \phi_{1}, \alpha_{c}\right) \simeq(1.150,0.769,1.797,1.230)$ and $(\delta u, \delta h, \delta a) \simeq$ $(0.147,-1.043,0.591) \delta \phi$. At the leading order there is a Lifshitz symmetry $\left(t, x, y, r^{-1}\right) \rightarrow$ $c\left(t, x, y, r^{-1}\right), z \rightarrow c^{\beta} z$ which can set $\delta \phi=-1$ to flow the Lifshitz geometry to $\mathrm{AdS}_{5}$. In the UV we have $(M / b)_{c} \simeq 0.986$. Note that for $q_{0}>0$ other relevant perturbations around the Lifshitz fixed point are always complex which indicates the ciritical point is unstable $[34,35]$ and we will confirm this by studying the free energy.

Integrating from the above near horizon solution to the boundary, we could obtain the full solution. Different from the previous studies on holographic semimetals $[9,15]$, we find that the near horizon behavior (2.18)-(2.21) flows to $M / b$ whose value runs from zero to $(M / b)_{c}$, and keeps increasing to a finite value of $(M / b)_{t+}$ with $(M / b)_{t+}>(M / b)_{c}$ and then turns back to $(M / b)_{c}$. While the near horizon behavior $(2.14)-(2.17)$ flows to $M / b$ whose value runs from infinity to $(M / b)_{c}$, and keeps decreasing to a finite $(M / b)_{t-}$ with $(M / b)_{t-}<(M / b)_{c}$ and increases to reach $(M / b)_{c}$ finally. ${ }^{7}$ Examples for the bulk profiles of

\footnotetext{
${ }^{7}$ Note that $(M / b)_{t+}=0.994,(M / b)_{t-}=0.977$ and $(M / b)_{c}=0.986$. These values can also be read from figure 2.
} 

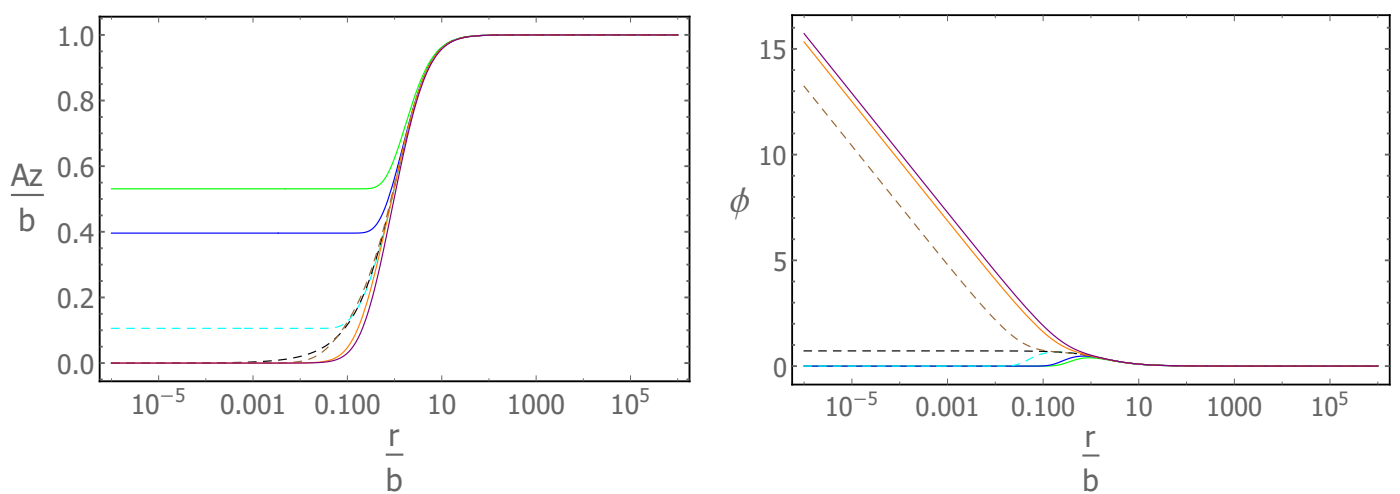

Figure 1. The bulk profiles of $A_{z}$ and $\phi$ for different values of $M / b=0.941$ (green), 0.983 (blue), 0.987 (dashed cyan), 0.986 (dashed black), 0.984 (dashed brown), 0.987 (orange), 1.019 (purple). The solid lines are profiles in the stable phase while dashed lines are for the unstable phase.

the matter fields at different values of $M / b$ are shown in figure 1 . The axial gauge field and the scalar field configurations in the topological phase and the insulating phase are generally separated by the bulk profiles (dashed black) at the critical point. In the topological phase, from UV to IR the axial gauge field $A_{z}$ decreases monotonically and ends at a finite value in the deep IR. The scalar field is not monotonic and it first increases, then decreases to zero in the deep IR. In the insulating phase, the axial gauge field decreases from UV to zero in the IR while the scalar field increases monotonically until it hits the IR singularity. Near the critical value of $M / b$, we observe oscillatory behavior of the matter fields (dashed color lines), which is due to the complex irrelevant deformations around the Lifshtiz fixed point. This can be taken as a signature of unstable critical solution, indicating that the phase transition is not continuous, which will be confirmed from the free energy in the next subsection.

\section{$2.2 \quad$ Asymptotic expansions and free energy}

In order to study the stability of the background, we shall study the free energy of the bulk geometry. The asymptotic behavior and free energy for the finite temperature case can be found in appendix B.2 and the zero temperature results can be obtained straightforwardly by setting $f=u$. At zero temperature, we have the following behaviour of fields near the UV boundary

$$
\begin{aligned}
u & =r^{2}-\frac{M^{2}}{6}+\frac{u_{2}}{r^{2}}+\ldots, \\
h & =r^{2}-\frac{M^{2}}{6}+\frac{b^{2} q_{0} M^{2}}{12} \frac{\log r}{r^{2}}+\frac{h_{2}}{r^{2}}+\ldots, \\
A_{z} & =b-\frac{b q_{0} M^{2}}{6} \frac{\log r}{r^{2}}+\frac{\eta}{r^{2}}+\ldots, \\
\phi & =\frac{M}{r}-\frac{b^{2} w_{0} M}{6} \frac{\log r}{r^{3}}+\frac{O}{r^{3}}+\ldots,
\end{aligned}
$$




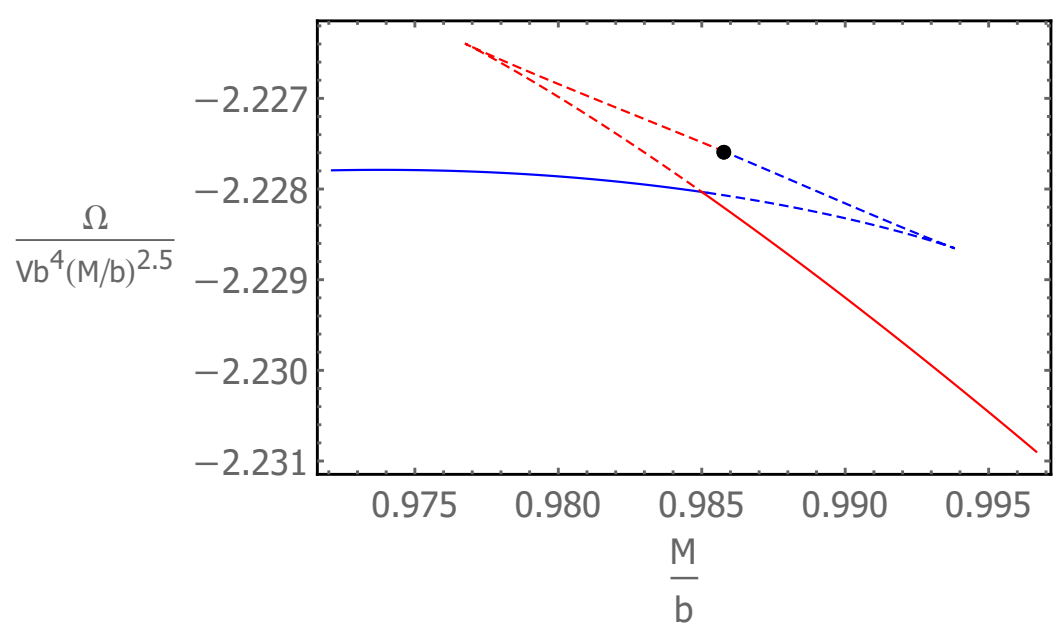

Figure 2. The free energy density as a function of $M / b$ for $q_{0}=15$. The blue (dashed) lines are solutions generated from the Weyl semimetal phase while the red (dashed) line are from the insulator phase. The black dot represents the free energy at the unstable critical point. The system undergoes a first order quantum phase transition from the Weyl semimetal phase to an insulating phase.

with $u_{2}=\frac{1}{6}(b \eta-M O)+\frac{1}{72} q_{0} b^{2} M^{2}+\frac{M^{4}}{108}$ and $h_{2}=-\frac{1}{3} b \eta-\frac{1}{6} M O-\frac{1}{144} q_{0} b^{2} M^{2}+\frac{M^{4}}{108}$. The free energy density can be obtained from the on-shell action to be

$$
\frac{\Omega}{V}=-\frac{1}{24} b^{2} M^{2} q_{0}-\frac{b \eta}{2}+\frac{M^{4}}{48}-\frac{M O}{2}
$$

where $\eta, O$ are the dual expectation values of the current and scalar operators. The free energy can also be written as $\frac{\Omega}{V}=\epsilon-T s$ with $\epsilon, s$ the energy and entropy densities (for details see appendix B.2).

With the bulk solution found in the previous subsection, we can obtain the free energy numerically. Figure 2 shows the free energy as a function of $M / b$ close to the phase transition. The critical point generated by IR geometry $(2.22)-(2.25)$ is unstable and the system undergoes a first order quantum phase transition from the Weyl semimetal phase to an insulator phase. ${ }^{8}$ This behavior exists for any $q_{0}>0 .{ }^{9}$ Notably this is quite different from the previous holographic model [9] in which a continuous holographic phase transition happens between the topological Weyl semimetal phase and a trivial semimetal phase. The different order of the phase transition may imply the different underlying mechanics for these two types of phase transitions.

The phase transitions for interacting Weyl semimetals were studied in [22] from the field theoretical approach, and it was found that for sufficiently strong interactions there exists a first order quantum phase transition between the Weyl semimetal and a normal band insulator. Our holographic study shows that the quantum phase transition from

\footnotetext{
${ }^{8}$ Note that these quantum phases are not distinguished by symmetry breaking.

${ }^{9}$ Following the rescaling mentioned in footnote 4 , one could recover the $\beta_{1}$ and $\beta_{2}$ by replacing $q_{0}$ by $q_{0} e_{2}$. Thus the order of the phase transition will not change for different values of $\beta_{1}$ and $\beta_{2}$. This is different from the well-known result in holographic p-wave superfluids studied in [25] in which the order of the phase transition depends on the ratio between gauge field coupling constant and gravitational coupling constant.
} 
strongly interacting Weyl semimetal to a Chern insulator (as we will show in the next section) is also of first order. Thus it broads our understanding on the phase structure of strongly interacting Weyl semimetals.

\section{Transport properties of the dual theory}

To figure out the exact nature of the stable phases, we should study the conductivities. In the following we will compute the full frequency dependent longitudinal and transverse electric conductivities. We will also study the phase diagram from the behavior of anomalous Hall conductivity at zero frequency.

The conductivities of a quantum many body system can be computed via the Kubo formula

$$
\sigma_{i j}=\lim _{\omega \rightarrow 0} \frac{1}{i \omega}\left\langle J_{i} J_{j}\right\rangle_{R}(\omega, \mathbf{k}=0) .
$$

In holography, the current-current retarded correlators can be computed by studying the fluctuations of the gauge fields dual to the currents around the background with infalling boundary conditions.

\subsection{Longitudinal conductivities}

We perturb the background (2.6) by the fluctuation $\delta V_{z}=v_{z}(r) e^{-i \omega t}$, and obtain the equation

$$
v_{z}^{\prime \prime}+\left(\frac{2 u^{\prime}}{u}-\frac{h^{\prime}}{2 h}+\frac{\partial_{\phi} Y}{Y} \phi^{\prime}\right) v_{z}^{\prime}+\frac{\omega^{2}}{u^{2}} v_{z}=0 .
$$

The electric conductivities depend on the form of dilatonic coupling $Y(\phi)$ in the action (2.1) which is chosen to be (2.12). Near the conformal boundary we have

$$
v_{z}=v_{z}^{(0)}+\frac{v_{z}^{(2)}}{r^{2}}+\frac{v_{z}^{(0)} \omega^{2} \log \Lambda r}{2 r^{2}}+\cdots
$$

With proper boundary conditions in the IR, the optical longitudinal conductivity is then

$$
\sigma_{z z}=\frac{1}{i \omega}\left(2 \frac{v_{z}^{(2)}}{v_{z}^{(0)}}-\frac{\omega^{2}}{2}\right)
$$

where we have considered the counterterm to cancel the $\log \Lambda r$ term.

In the phase with IR geometry (2.14)-(2.17), there are two linearly independent solutions for $v_{z}$ in IR

$$
\begin{aligned}
& v_{z 1} \simeq c_{1} r^{\frac{1}{4}\left(1+\sqrt{1-16 \omega^{2}}\right)}(1+\mathcal{O}(r)) \\
& v_{z 2} \simeq c_{2} r^{\frac{1}{4}\left(1-\sqrt{1-16 \omega^{2}}\right)}(1+\mathcal{O}(r)) .
\end{aligned}
$$

Both these two solutions are real and normalisable when $\omega<\Delta=1 / 4$. The unique boundary condition can be fixed by the analyticity condition of the correlator in $\omega$ [26]. 
When $\omega>\Delta$, the solutions become complex and we can choose the infalling boundary condition

$$
v_{z} \simeq r^{\frac{1}{4}\left(1-i \sqrt{16 \omega^{2}-1}\right)}(1+\mathcal{O}(r)) .
$$

The solution in (3.5) and (3.6) to produce the above infalling boundary conditon under the $\omega \rightarrow \omega+i \epsilon$ prescription is the first one, i.e. we choose the boundary condition for $v_{z}$ when $\omega<\Delta$

$$
v_{z} \simeq r^{\frac{1}{4}\left(1+\sqrt{1-16 \omega^{2}}\right)}(1+\mathcal{O}(r)) .
$$

Since this boundary condition is real for $\omega<\Delta$, this leads to the result that the real part of the conductivity $\sigma_{z z}(\omega)$ vanishes. There is a continuum above a gap in the optical longitudinal conductivity. Note that in this calculation we have set the unit in which the IR horizon geometry is of the form (2.14)-(2.17). As this solution flows to a specific value of $A_{z} \simeq b_{0}$ at the UV boundary, this indicates that if we set the unit $b=1$, the width of the hard gap $\Delta / b$ is $1 / 4 b_{0}$. As $b_{0}$ depends on the parameter $a_{1}$ in (2.16) which will generate different $M / b$, we shall have different width of the gap for different $M / b$ in the insulating phase. Another equivalent way to see that there is indeed a hard gap in the optical conductivity is from the Schrodinger potential approach in appendix C.

In the phase with IR geometry (2.18)-(2.21), the IR infalling boundary condition for $v_{z}$ is

$$
v_{z} \simeq \frac{-i \omega}{r} K_{1}\left[\frac{-i \omega}{r}\right]
$$

In this case there is always a continous gapless spectrum for $\operatorname{Re}\left[\sigma_{z z}(\omega)\right]$.

With the boundary conditions (3.7) and (3.9), we could obtain the retarded Green function. In figure 3, we plot the real part of the longitudinal conductivity in the topological phase and the insulating phase for different values of $M / b$. In the topological phase, the longitudinal conductivity is linear in $\omega$ at both small and large frequency regimes, which is similar to the holographic results in [17]. In the insulating phase there is a hard gap in the conductivity which confirms the nature of holographic insulating phase. There exists a continuum gapless spectrum above the gap and the conductivity eventually becomes linear in $\omega$ at large frequency.

The dependance of the width of the hard gap as a function of $M / b$ is shown in figure 4 . Similar to the weakly coupled case, it is monotonically increasing when we increase $M / b$ in the insulator phase and for large enough $M / b$, we have $\Delta / b \propto 0.22(M / b-0.3)$.

\subsection{Transverse conductivities}

The transverse conductivities can be studied by considering fluctuations $\delta V_{x}=$ $v_{x}(r) e^{-i \omega t}, \delta V_{y}=v_{y}(r) e^{-i \omega t}$. The corresponding equations for $v_{x}$ and $v_{y}$ are

$$
\begin{aligned}
& v_{x}^{\prime \prime}+\left(\frac{u^{\prime}}{u}+\frac{h^{\prime}}{2 h}+\frac{\partial_{\phi} Y}{Y} \phi^{\prime}\right) v_{x}^{\prime}+\frac{\omega^{2}}{u^{2}} v_{x}+8 i \alpha \omega \frac{A_{z}^{\prime}}{Y u \sqrt{h}} v_{y}=0 \\
& v_{y}^{\prime \prime}+\left(\frac{u^{\prime}}{u}+\frac{h^{\prime}}{2 h}+\frac{\partial_{\phi} Y}{Y} \phi^{\prime}\right) v_{y}^{\prime}+\frac{\omega^{2}}{u^{2}} v_{y}-8 i \alpha \omega \frac{A_{z}^{\prime}}{Y u \sqrt{h}} v_{x}=0
\end{aligned}
$$




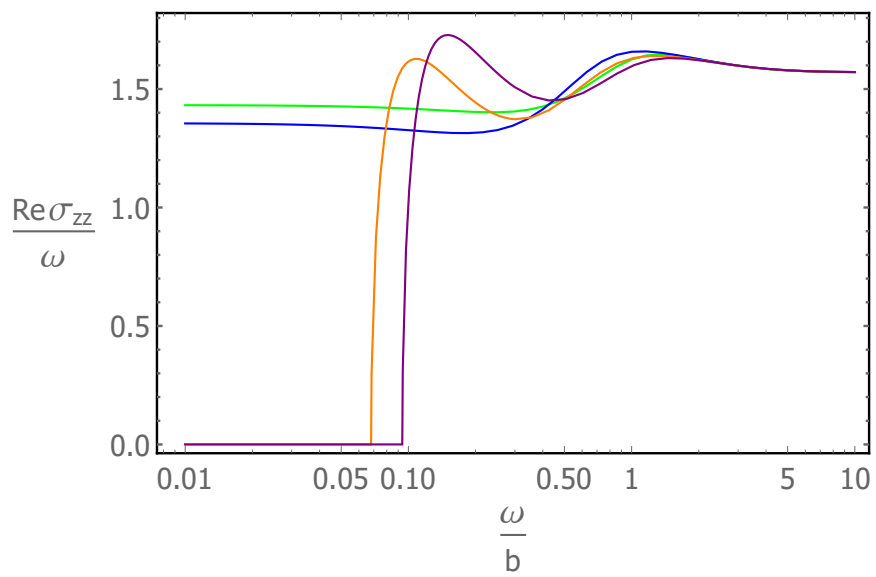

Figure 3. The real part of longitudinal conductivity as a function of the frequency $\omega / b$ for different values of $M / b=0.941$ (green), 0.983 (blue), 0.987 (orange), 1.019 (purple) in the topological phase and the insulating phase.

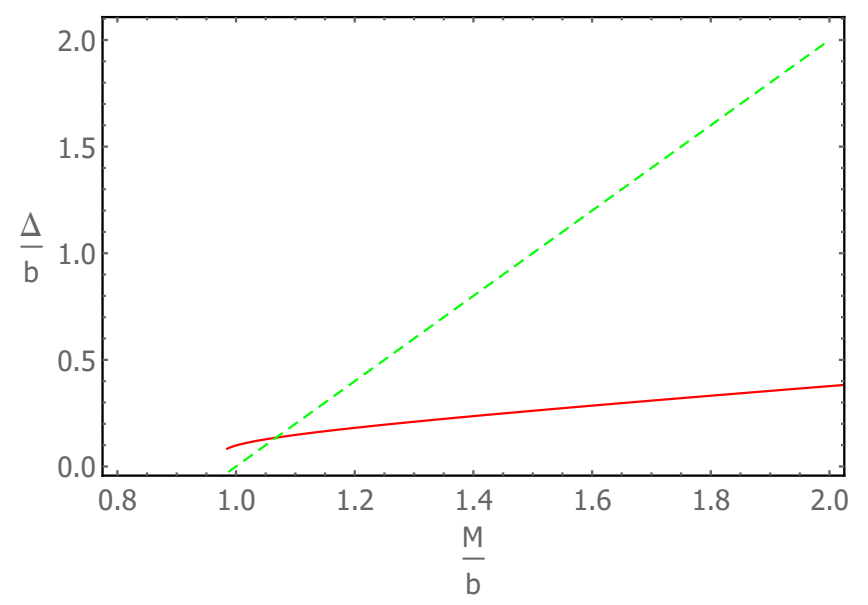

Figure 4. The dependance of the width of the hard gap as a function of $M / b$ in the holographic insulating phase (red) and weakly coupled field theory (dashed green).

Define $v_{ \pm}=v_{x} \pm i v_{y}$, we obtain

$$
v_{ \pm}^{\prime \prime}+\left(\frac{u^{\prime}}{u}+\frac{h^{\prime}}{2 h}+\frac{\partial_{\phi} Y}{Y} \phi^{\prime}\right) v_{ \pm}^{\prime}+\frac{\omega^{2}}{u^{2}} v_{ \pm} \pm 8 \alpha \omega \frac{A_{z}^{\prime}}{Y u \sqrt{h}} v_{ \pm}=0
$$

For the last two terms, in the deep IR $r \rightarrow 0$, the term $\frac{\omega^{2}}{u^{2}}$ always dominates. Thus the near horizon boundary conditions are the same as the case for the longitudinal conductivities. More explicitely, for IR geometry (2.18)-(2.21), the IR infalling boundary conditions $v_{ \pm}=\frac{-i \omega}{r} K_{1}\left[\frac{-i \omega}{r}\right]$, while for IR geometry (2.14)-(2.17), the IR boundary conditions are $v_{ \pm} \simeq r^{\frac{1}{4}\left(1+\sqrt{1-16 \omega^{2}}\right)}(1+\mathcal{O}(r))$ when $\omega<\Delta=1 / 4$ and $v_{ \pm} \simeq r^{\frac{1}{4}\left(1-i \sqrt{16 \omega^{2}-1}\right)}(1+\mathcal{O}(r))$ when $\omega>\Delta$.

With the Green functions $G_{ \pm}$from the new variables $v_{ \pm}$, we can compute $G_{x x}, G_{y y}$ and $G_{x y}$. We have $\sigma_{x y} \pm i \sigma_{x x}= \pm \frac{G_{ \pm}}{\omega}$, i.e.

$$
\sigma_{T}=\sigma_{x x}=\sigma_{y y}=\frac{G_{+}+G_{-}}{2 i \omega}, \quad \sigma_{x y}=\frac{G_{+}-G_{-}}{2 \omega} .
$$



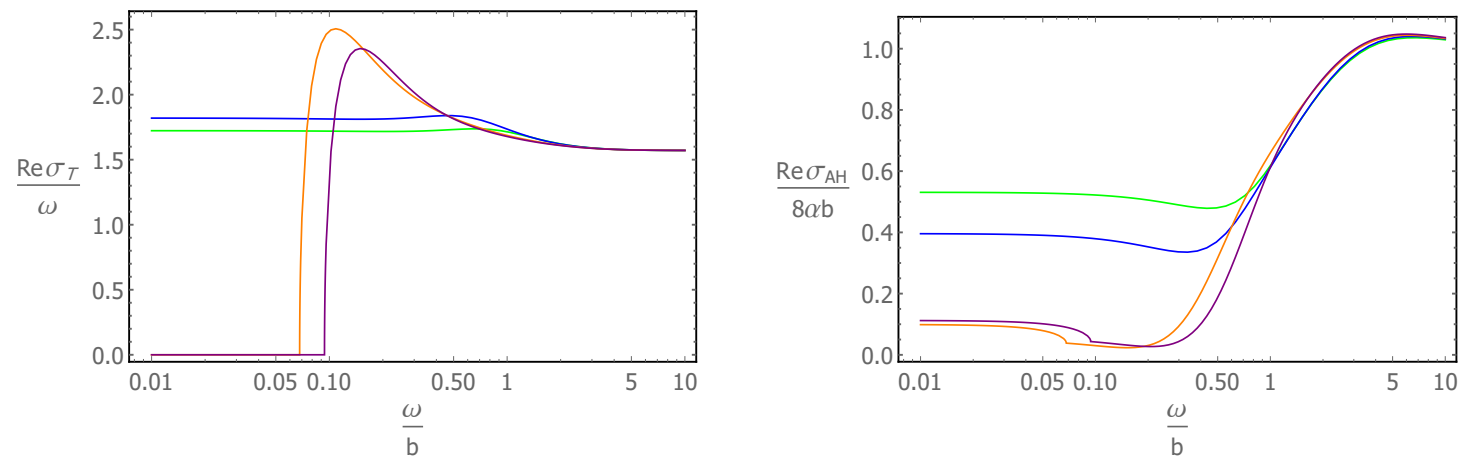

Figure 5. The real part of the transverse conductivity (left) and the anomalous Hall conductivity (right) as a function of frequency at different values of $M / b=0.941$ (green), 0.983 (blue), 0.987 (orange), 1.019 (purple) in the topological phase and the insulator phase.

The Chern-Simons term in the consistent current contributes to the anomalous Hall conductivity. We define $\mathbf{J}_{\text {cons }}=\sigma_{\mathrm{AH}} \mathbf{e}_{\mathbf{b}} \times \mathbf{E}$ and we have $\sigma_{\mathrm{AH}}=8 \alpha b-\sigma_{x y}=8 \alpha b-\frac{G_{+}-G_{-}}{2 \omega}[10]$.

The numerical results for the full frequency dependent transverse conductivities are shown in figure 5 . The left plot in figure 5 is for the real part of optical transverse conductivities $\sigma_{x x}$ and $\sigma_{y y}$. We have a gapless spectrum in the Weyl semimetal phase. In the insulating phase, there is a continuous gapless spectrum above a hard gap $\Delta / b=1 / 4 b_{0}$. This behavior is similar to the longitudinal component. Different from the longitudinal one, in the Weyl semimetal phase if we increase $M / b$, the ratio of the transverse conductivity $\operatorname{Re} \sigma_{T}$ to the frequency increases at low frequency. This difference is caused by the emergent Lifshitz symmetry in the critical point which leads to the result that $\operatorname{Re}\left[\sigma_{z z}(\omega)\right] \propto \omega^{2-\beta}$ while both $\operatorname{Re}\left[\sigma_{T}(\omega)\right]$ and $\operatorname{Re}\left[\sigma_{\mathrm{AH}}(\omega)\right]$ are proportional to $\omega^{\beta}$ when $M / b$ is approaching the (unstable) critical value $[16,17]$. The right plot in figure 5 is the real part of optical anomalous Hall conductivity. In the insulating phase different from the diagonal component, the anomalous Hall conductivity approaches a nonzero value at zero frequency although there is an emergent time reversal symmetry in the deep IR. This is because $\sigma_{\mathrm{AH}}$ depends on the real part of $G_{ \pm}$, it is nonvanishing when $\omega<\Delta$ and there is no hard gap. The nonvanishing $\sigma_{\mathrm{AH}}$ crucially depends on the IR boundary condition for the fluctuations which is fixed by the $\omega \rightarrow \omega+i \epsilon$ prescription. Furthermore, we observe that there is smooth change at $\omega=\Delta$ for the optical anomalous Hall conductivity in the insulating phase.

The behavior of conductivities in the insulating phase resembles that of a Chern insulator, indicating that our holographic model realises a quantum phase transition from a topological Weyl semimetal to a Chern insulator. In weakly coupled field theory, there are models to describe the phase transition from a Weyl semimetal to a Chern insulator $[21,23,24]$. Our holographic study confirms that a similar phase structure exists for strongly interacting Weyl semimetal.

\subsection{Phase diagram}

The order parameter of the quantum phase transition between the topological phase and the insulating phase is the DC anomalous Hall conductivity. The DC anomalous Hall 
conductivities can be computed using a near-far matching method following [9]. We show here the explicit procedure of the calculations in the topological phase, and also comment on the calculations in the insulating phase.

In the topological phase, near horizon the solution of $v_{ \pm}$with infalling boundary condition is $v_{ \pm}^{(n 0)}=\frac{-i \omega}{r} K_{1}\left[\frac{-i \omega}{r}\right]$. In the matching regime $\omega \ll r \ll \min \{M, b\}$, this solution can be expanded as

$$
v_{ \pm}^{(n 0)}=1-\frac{\omega^{2}}{4 r^{2}}\left(-1+2 \gamma+2 \ln \left[\frac{-i \omega}{2 r}\right]\right),
$$

where $\gamma$ is the Euler-Mascheroni constant. From this expansion, we know that at the matching region the infalling solution corresponds to the solution 1 while the $\omega^{2}$ term can be ignored since we are interested in the $\omega \rightarrow 0$ result. The linear order correction in $\omega$ to the near region solution is sourced by the infalling leading order solution. Thus at matching region the full linear order in $\omega$ boundary condition is

$$
v_{ \pm}^{(n)}=1+\omega v_{ \pm}^{(n 1)}
$$

where $v_{ \pm}^{(n 1)^{\prime}}=\mp \frac{8 \alpha\left(A_{z}(r)-A_{z}(0)\right)}{r^{3}}$.

In the far region $\omega \ll r$, we have

$$
v_{ \pm}^{(f)^{\prime \prime}}+\left(\frac{h^{\prime}}{2 h}+\frac{u^{\prime}}{u}+\frac{\partial_{\phi} Y}{Y} \phi^{\prime}\right) v_{ \pm}^{(f)^{\prime}} \pm \frac{8 \omega \alpha}{Y u \sqrt{h}} A_{z}^{\prime} v_{ \pm}^{(f)}+\frac{\omega^{2}}{u^{2}} v_{ \pm}^{(f)}=0
$$

Its solution can be expanded according to $\omega$ and we will solve the equation (3.16) up to the first order in $\omega$. Note that the last term in (3.16) can be ignored at order $\omega$. With the near horizon boundary condition (3.15), we obtain the solution $v_{ \pm}^{(f)}=1+\omega v_{ \pm}^{(f 1)}$ where $v_{ \pm}^{(f 1)^{\prime}}=\mp \frac{8 \alpha\left(A_{z}(r)-A_{z}(0)\right)}{Y u \sqrt{h}}$.

With the far region solutions, we obtain $G_{ \pm}=\omega\left( \pm 8 \alpha\left(b-A_{z}(0)\right)\right)$. From (3.13) we obtain the DC conductivities

$$
\sigma_{x y}=\frac{G_{+}-G_{-}}{2 \omega}=8 \alpha\left(b-A_{z}(0)\right), \quad \sigma_{x x}=\sigma_{y y}=0 .
$$

Note that in the computations above, the result of (3.17) is for the anomalous Hall conductivity defined from the covariant currents. In the following, we will obtain the anomalous Hall conductivity for the consistent currents which is more close to results in real experimental systems $[4,10]$. The final result for the zero frequency anomalous Hall conductivity in the holographic Weyl semimetal phase is

$$
\sigma_{\mathrm{AHE}}=\operatorname{Re}\left[\sigma_{\mathrm{AH}}(\omega \rightarrow 0)\right]=8 \alpha b-\sigma_{x y}=8 \alpha A_{z}(0)
$$

which is completely determined by the near horizon value of the axial gauge field.

In the gapped phase, one could attempt to repeat the above near-far matching method to compute the DC anomalous Hall conductivity. The solution at the matching regime should be modified to be $v_{ \pm}^{(n)}=\sqrt{r}+\omega v_{ \pm}^{(n 1)}$, which leads to the observation that $\omega v_{ \pm}^{(n 1)}$ can not be determined analytically. In the insulating phase we do not have a simple formula 

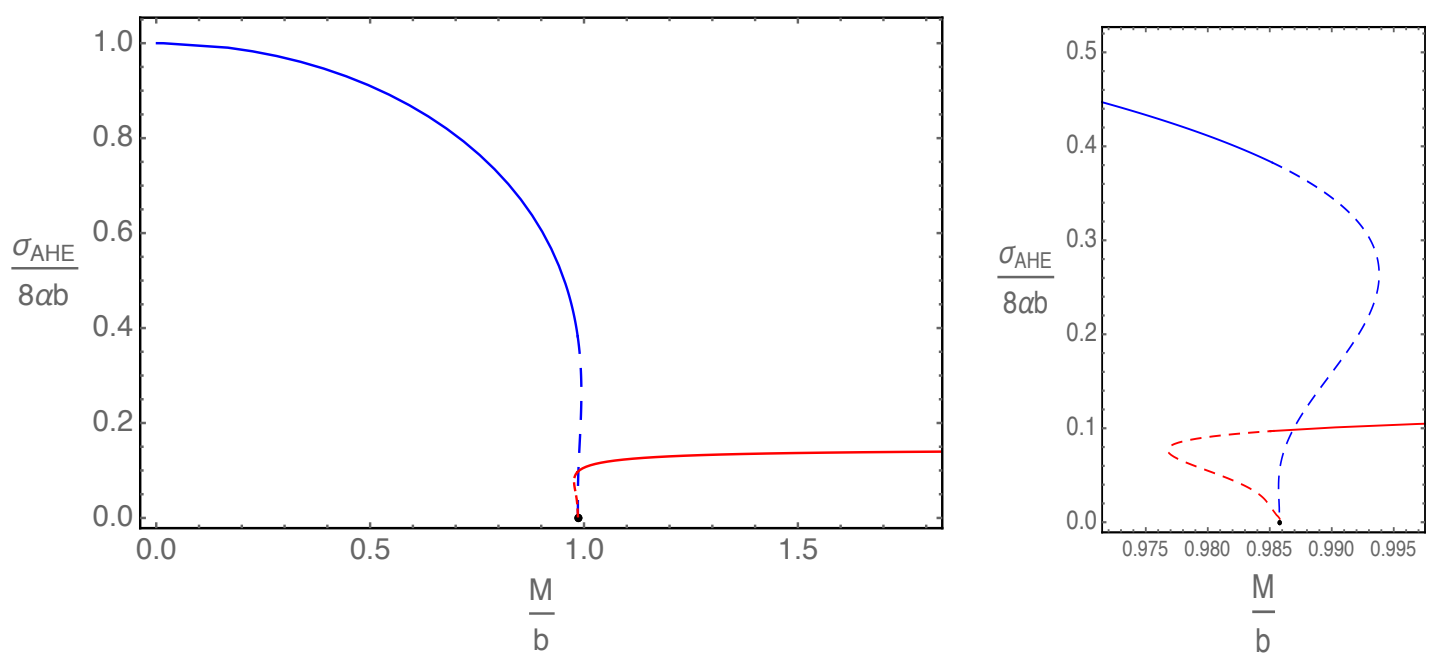

Figure 6. Both plots are for the zero temperature anomalous Hall conductivity at zero frequency of the holographic system. The right plot is a zoomed in version of the left plot close to the phase transiton point. In both plots, the blue line is for the topological Weyl semimetal phase and the red line is for the Chern insulator phase. The solid and dashed lines are for stable and unstable phases respectively. We see that under the phase transition, the anomalous Hall conductivity is discontinuous and the system undergoes a first order phase transition from a Weyl semimetal phase to a Chern insulator phase.

as (3.18). This is also intuitively correct. The boundary condition (3.8) is determined by the analytical continuation of the large frequency condition which reflects necessary information beyond the near horizon behavior. Therefore we have to compute the DC anomalous Hall conductivity numerically by taking $\omega \rightarrow 0$ limit of $\operatorname{Re}\left[\sigma_{\mathrm{AH}}(\omega)\right]$ obtained from the last subsection.

Figure 6 shows the anomalous Hall conductivity at zero frequency as a function of $M / b$. The solid line is for the stable phase while the dashed line is for the unstable phase, similar to the free energy plots in figure 2 . This figure shows that when we increase $M / b$ the nonzero anomalous Hall conductivity decreases and jump directly at a phase transition point to a nonzero value which seems to be insensitive to $M / b$. The blue lines is for the background from IR geometry (2.18)-(2.21) while the red line is for the solutions from (2.14)-(2.17). The discontinuity of the zero frequency anomalous Hall conductivity further supports that this holographic phase transiton is of first order, which is consistent with the result from the free energy analysis. Moreover, in the holographic insulating phase, in the diagonal components of the optical conductivities there is a continuous gapless spectrum above a hard gap, and the zero frequency anomalous Hall conductivity is nonzero. These are the signals of a Chern insulator. Therefore, our holographic model describes a first order quantum phase transition from a strongly interacting Weyl semimetal to a Chern insulator.

\section{Conclusion and discussion}

We have provided a holographic model to charaterize the quantum phase transition between the strongly interacting Weyl semimetal and the Chern insulator, by tunning the ratio 
between the mass parameter and time reversal symmetry breaking parameter in the dual field theory. We established that this quantum phase transition is of first order. We also computed the frequency dependent conductivities numerically in each phase. In the holographic Weyl semimetal phase, we found that there is a nontrivial DC anomalous Hall conductivity and the diagonal components of optical conductivities are linear in frequency in both small and large frequency regimes. In the holographic Chern insulator phase, we found that there is a hard gap in the real part of the diagonal components of the frequency dependent conductivities and there is also a nonvanishing DC anomalous Hall conductivity. This is a first example of Chern insulator from holography signified by a nontrivial anomalous Hall conductivity in a gapped state.

Our holographic model reveals the interesting phase diagram for strongly interacting Weyl semimetal and provides a novel framework to explore further problems of strongly coupled topological states. There are many open questions. Firstly, in the particular holographic model we studied, the phase transition is of first order. It would be interesting to see if it is still first order for more general holographic phase transition models between Weyl semimetal and insulating phase with different dilatonic couplings. It is also important to have a better understanding of the essential physics at the first order holographic quantum phase transition point. Secondly, note that in field theory, there are studies of the disorder effects on the quantum phase transitions between Weyl semimetal and Chern insulators [23, 24, 39]. It would be worthwhile to explore the disorder effects or other momentum dissipation effects on this holographic quantum phase transition to understand the similarities or differences to the weakly coupled field theoretical results. Meanwhile, the transport properties of the holographic system at finite temperature is to be further explored. Finally, the insulating phase we found in this work is a Chern insulator with nontrivial anomalous Hall conductivity. It would be very interesting to study the topological invariants of this holographic Chern insulator following [14], to explore effects of surface states, to realise the phase transition to a normal insulator and so on. These studies should be helpful to build holographic models for topological insulators towards more complicated topological states of mater. We hope to explore some of these questions further in the future.

\section{Acknowledgments}

We thank Rong-Gen Cai, Karl Landsteiner, Francisco Pena-Benitez, Jie Ren, Shun-Qing Shen, Ya-Wen Sun, Zhong Wang for helpful discussions. This work was supported by the National Thousand Young Talents Program of China, NFSC Grant No.11875083 and a grant from Beihang University. Y.L. would also like to thank Hanyang University for the hospitality during the APCTP focus program "Holography and Geometry of Quantum Entanglement" where this work was presented. 


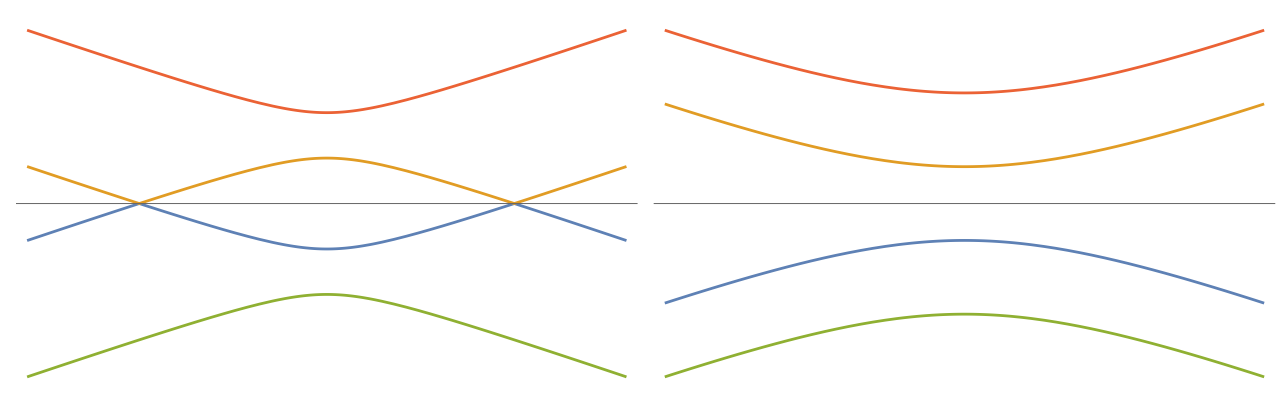

Figure 7. The energy spectrum as a function of $k_{z}$ in the weakly coupled theory. Left: when $M<b$, there are Weyl points separating by an effective separation $2 \sqrt{b^{2}-M^{2}}$ which indicates the system is in the Weyl semimetal phase. Right: when $M>b$, there is a band gap with $2(M-b)$ in the spectrum and the system is in an insulating phase.

\section{A Weakly coupled field theory for Weyl semimetal}

In this appendix we briefly review the weakly coupled field theory model for Weyl semimetals. A simple low energy effective theory for Weyl semimetal [36-38] is

$$
\mathcal{L}=i \bar{\psi}\left[\gamma^{\mu}\left(\partial_{\mu}-i e A_{\mu}\right)-\gamma_{5} \gamma^{\mu} b_{\mu}\right] \psi+M \bar{\psi} \psi
$$

Here $b_{0}$ plays the role of axial chemical potential which breaks inversion symmetry, while $b_{i}$ plays the role of separation which breaks time reversal symmetry. We chose $b_{\mu}=b \delta_{\mu}^{z}$. The spectrum can be computed as $E_{i j}=(-1)^{i} \sqrt{k_{x}^{2}+k_{y}^{2}+\left(b+(-1)^{j} \sqrt{M^{2}+k_{z}^{2}}\right)^{2}}$ with $(i, j)=1,2$. The energy spectrum as a function of $k_{z}$ while $k_{x}=k_{y}=0$ is shown in figure 7 from which it is clear that tunning $M / b$ the system undergoes a quantum phase transition from the Weyl semimetal to a normal band insulator. This topological phase transition can also be characterized by the DC anomalous Hall conductivity. More precisely, in the Weyl semimetal phase we have $\sigma_{\mathrm{AHE}}=\frac{1}{2 \pi^{2}} \sqrt{b^{2}-M^{2}}$ while in the insulating phase we have $\sigma_{\mathrm{AHE}}=0$. It is also interesting to note that a similar weakly coupled field theory exists for other topological semimetals, e.g. for nodal line semimetal [3, 15].

The Ward identity of the weakly coupled theory (A.1) is

$$
\nabla_{\mu} J^{\mu}=0, \quad \nabla_{\mu} J_{5}^{\mu}=\frac{1}{16 \pi^{2}} \epsilon^{\mu \nu \rho \lambda} F_{\mu \nu} F_{\rho \lambda}+M \bar{\psi} \gamma^{5} \psi
$$

Notably the holographic model in the main text produced exactly the same Ward identity.

It is also possible to construct field theory model from a Weyl semimetal to a Chern insulator (3+1D anomalous Hall state) [21, 23, 24, 39, 40]. For example, if we put the above model in a lattice, by tunning $M / b$ to make the Weyl nodes located at the Brillouin zone boundary to be annihilated pairwise, the system shows a quantum phase transition from the Weyl semimetal to a Chern insulator. In the Chern insulator phase $(3+1 \mathrm{D}$ anomalous Hall state), the longitudinal and transverse conductivities are gapped while there is a nontrivial DC anomalous Hall conductivity. The holographic model studied in the main text shows similar behaviors in the topological trivial phase after the phase transition and it is tempting to identify this phase as a holographic Chern insulator phase. 


\section{B Equations of motion at finite temperature}

In the main text we focus on the zero temperature case and for completeness we list the calculations for finite temperature in this appendix. The ansatz for the background fields at finite temperature is

$$
d s^{2}=-u d t^{2}+\frac{d r^{2}}{u}+f\left(d x^{2}+d y^{2}\right)+h d z^{2}, \quad A=A_{z} d z, \quad \phi=\phi(r),
$$

where fields $u, f, h, A_{z}, \phi$ are functions of the radial coordinate $r$.

Plugging the ansatz (B.1) into equation, we obtain the equations of motion

$$
\begin{array}{r}
\frac{f^{\prime \prime}}{f}-\frac{u^{\prime \prime}}{u}+\frac{f^{\prime} h^{\prime}}{2 f h}-\frac{h^{\prime} u^{\prime}}{2 h u}=0, \\
\frac{f^{\prime \prime}}{f}+\frac{u^{\prime \prime}}{2 u}-\frac{f^{\prime 2}}{4 f^{2}}+\frac{f^{\prime} u^{\prime}}{f u}-\frac{6}{u}+\frac{V}{2 u}-\frac{W A_{z}^{2}}{4 h u}-\frac{Z A_{z}^{\prime 2}}{4 h}+\frac{\phi^{\prime 2}}{4}=0, \\
\frac{1}{4} \phi^{\prime 2}+\frac{6}{u}-\frac{u^{\prime}}{2 u}\left(\frac{f^{\prime}}{f}+\frac{h^{\prime}}{2 h}\right)-\frac{f^{\prime} h^{\prime}}{2 f h}-\frac{f^{\prime 2}}{4 f^{2}}-\frac{V}{2 u}-\frac{W A_{z}^{2}}{4 u h}+\frac{Z A_{z}^{\prime 2}}{4 h}=0, \\
A_{z}^{\prime \prime}+A_{z}^{\prime}\left(\frac{f^{\prime}}{f}-\frac{h^{\prime}}{2 h}+\frac{u^{\prime}}{u}+\frac{\phi^{\prime} \partial_{\phi} Z}{Z}\right)-\frac{A_{z} W}{u Z}=0, \\
\phi^{\prime \prime}+\phi^{\prime}\left(\frac{f^{\prime}}{f}+\frac{h^{\prime}}{2 h}+\frac{u^{\prime}}{u}\right)-\frac{\partial_{\phi} V}{u}-\frac{A_{z}^{2} \partial_{\phi} W}{2 h u}-\frac{A_{z}^{\prime 2} \partial_{\phi} Z}{2 h}=0,
\end{array}
$$

where the prime is the derivative with respect to the radial coordinate $r$. The first equation can be written as $\left(\sqrt{h}\left(u f^{\prime}-u^{\prime} f\right)\right)^{\prime}=0$ which is essentially a conserved Noether charge. Note that setting $f=u$, we get the equations of motion for the zero temperature case, i.e. the equation (B.2) is trivial and we reduced to the four independent ODEs (2.7)-(2.10) in the main text.

There is another conserved Noether charge

$$
J^{r}=2 u^{5 / 2}\left(\frac{f \sqrt{h}}{u^{3 / 2}}\right)^{\prime}+\frac{u f}{\sqrt{h}} Z A_{z} A_{z}^{\prime}
$$

satisfying $\partial_{r} J^{r}=0$ associated with the following scaling symmetry $(x, y, z) \rightarrow$ $c(x, y, z), t \rightarrow t / c^{3}, r \rightarrow c^{3} r, u \rightarrow c^{6} u,(f, h) \rightarrow(f, h) / c^{2}, A_{z} \rightarrow A_{z} / c, \phi \rightarrow \phi$. This Noether charge is useful to check the accuracy of numerical code. Meanwhile, the following scaling symmetries are useful to work in certain unit, e.g. $b=1$ for zero temperature case.

(I.) $r \rightarrow \lambda r, \quad(t, x, y, z) \rightarrow \lambda^{-1}(t, x, y, z), \quad(u, f, h) \rightarrow \lambda^{2}(u, f, h), \quad A_{z} \rightarrow \lambda A_{z}$;

(II.) $(x, y) \rightarrow \lambda(x, y), \quad f \rightarrow \lambda^{-2} f$;

(III.) $z \rightarrow \lambda z, \quad h \rightarrow \lambda^{-2} h, \quad A_{z} \rightarrow \lambda^{-1} A_{z}$.

We can use the first scaling symmetry to make the black hole horizon located at $r_{0}=1$ in the finite temperature case. The last two symmetry can further take the leading asymptotic coefficients of $f$ and $h$ to be 1 . 


\section{B.1 Near horizon}

Near horizon $r \rightarrow r_{0}$, we have the expansion

$$
\begin{aligned}
u & =4 \pi T\left(r-r_{0}\right)+\ldots, \\
f & =f_{1}+\frac{f_{1} A_{z 2}\left(3+10 e^{\sqrt{2 / 3} \phi_{1}}+3 e^{2 \sqrt{2 / 3} \phi_{1}}\right)}{A_{z 1} q_{0}\left(e^{\sqrt{2 / 3} \phi_{1}}-1\right)^{2}}\left(r-r_{0}\right)+\ldots, \\
h & =h_{1}+\ldots, \\
A_{z} & =A_{z 1}+A_{z 2}\left(r-r_{0}\right)+\ldots, \\
\phi & =\phi_{1}-\frac{A_{z 2}\left(e^{\sqrt{2 / 3} \phi_{1}}+1\right)\left(9 h_{1}-A_{z 1}^{2} q_{0}\right)}{\sqrt{6} h_{1} A_{z 1} q_{0}\left(e^{\sqrt{2 / 3} \phi_{1}}-1\right)}\left(r-r_{0}\right)+\ldots,
\end{aligned}
$$

with $T=\frac{A_{z 1} q_{0} e^{-\sqrt{2 / 3} \phi_{1}}\left(e^{\sqrt{2 / 3} \phi_{1}}-1\right)^{2}}{8 \pi A_{z 2}}$. The independent parameters are $T, f_{1}, h_{1}, A_{z 1}, \phi_{1}$. With the above scaling symmetries, we only have two free parameters, which correspond to $M / b, T / b$ in the dual field theory.

\section{B.2 Asymptotic behavior and free energy}

Close to the UV boundary (i.e. $r \rightarrow \infty$ ), we obtain the following behaviour of fields

$$
\begin{aligned}
u & =r^{2}-\frac{M^{2}}{6}+\frac{u_{2}}{r^{2}}+\ldots, \\
f & =r^{2}-\frac{M^{2}}{6}+\frac{f_{2}}{r^{2}}+\ldots, \\
h & =r^{2}-\frac{M^{2}}{6}+\frac{b^{2} q_{0} M^{2}}{12} \frac{\log r}{r^{2}}+\frac{h_{2}}{r^{2}}+\ldots, \\
A_{z} & =b-\frac{b q_{0} M^{2}}{6} \frac{\log r}{r^{2}}+\frac{\eta}{r^{2}}+\ldots, \\
\phi & =\frac{M}{r}-\frac{b^{2} q_{0} M}{6} \frac{\log r}{r^{3}}+\frac{O}{r^{3}}+\ldots,
\end{aligned}
$$

with $h_{2}=-2 f_{2}-\frac{M O}{2}+\frac{1}{48} b^{2} q_{0} M^{2}+\frac{M^{4}}{36}$. Furthermore, one can obtain $f_{2}=u_{2}+\pi T f_{1} \sqrt{h_{1}}$ and $h_{2}=u_{2}-\frac{1}{2} b \eta-\frac{1}{48} b^{2} q_{0} M^{2}+\pi T f_{1} \sqrt{h_{1}}$ from the two conserved Noether charges evaluated at the horizon and conformal boundary. These relations show that $u_{2}, f_{2}, h_{2}$ can be fully determined by $b, M, \eta, O, T f_{1} \sqrt{h_{1}}$. Note that one can determine the above expansions only up to a shift $r \rightarrow r+a$. It is worth to point out that different from the minimal model in [9], we do not have any correction of order $\frac{\log r}{r^{2}}$ in $u$ and $f$.

To compute the free energy, we need to obtain the on-shell action. The renormalised action is

$$
S_{\text {ren }}=S+S_{\mathrm{GH}}+S_{\text {c.t. }}
$$

where the Gibbons-Hawking term is

$$
S_{\mathrm{GH}}=\frac{1}{\kappa^{2}} \int d^{4} x \sqrt{-\gamma} K
$$


and the counterterm

$$
\begin{aligned}
S_{\text {c.t. }}= & \frac{1}{2 \kappa^{2}} \int d^{4} x \sqrt{-\gamma}\left[-6-\frac{\phi^{2}}{2}\right] \\
& +\log r \int d^{4} x \sqrt{-\gamma}\left[\frac{1}{4} \mathcal{F}_{\mu \nu} \mathcal{F}^{\mu \nu}+\frac{1}{4} F_{\mu \nu} F^{\mu \nu}+\frac{1}{2}\left(\partial_{\mu} \phi\right)^{2}+\frac{W(\phi)}{2} A_{\mu}^{2}\right] .
\end{aligned}
$$

Note that $\gamma_{a b}=g_{a b}-n_{a} n_{b}$ is the induced metric on the boundary surface $r=r_{\infty}$ with $n^{a}$ the outward unit vector normal to the boundary. The trace of the extrinsic curvature is $K=\gamma^{a b} \nabla_{a} n_{b}$.

For the ansatz (B.1), the renormalized on-shell action is

$$
\frac{S_{\mathrm{os}}}{V}=\frac{1}{24} b^{2} M^{2} q_{0}+\frac{b \eta}{2}+\pi T f_{1} \sqrt{h_{1}}-\frac{M^{4}}{48}+\frac{M O}{2},
$$

therefore the free energy of the system is

$$
\frac{\Omega}{V}=-\frac{S_{\mathrm{os}}}{V}=-\frac{1}{24} b^{2} M^{2} q_{0}-\frac{b \eta}{2}-\pi T f_{1} \sqrt{h_{1}}+\frac{M^{4}}{48}-\frac{M O}{2} .
$$

The thermodynamics of the dual system can be obtained straightforwardly. The expectation value of the stress tensor can be computed from

$$
T_{\mu \nu}=2\left(K_{\mu \nu}-\gamma_{\mu \nu} K\right)+\frac{2}{\sqrt{-\gamma}} \frac{\delta S_{\text {c.t. }}}{\delta \gamma^{\mu \nu}} .
$$

We obtain $\epsilon=-\frac{1}{24} b^{2} M^{2} q_{0}-\frac{b \eta}{2}+3 \pi T f_{1} \sqrt{h_{1}}+\frac{M^{4}}{48}-\frac{M O}{2}$. Thus the free energy density is $\frac{\Omega}{V}=\epsilon-4 \pi T f_{1} \sqrt{h_{1}}=\epsilon-T s$ where $s$ is the entropy density expressed in unit $2 \kappa^{2}=1$.

\section{Schrodinger potential approach to conductivities}

The retarded Green's function can be computed from another equivalent approach which was widely studied in e.g. [26, 32]. The idea is to transform the equation of motion for the fluctuations into a Schrodinger potential problem. In the following we shall assume in the IR at the leading order the geometry is of a generic form ${ }^{10}$

$$
u \simeq u_{0} r^{\alpha_{1}}, \quad h \simeq h_{0} r^{\alpha_{2}}, \quad A_{z} \simeq a_{1} r^{\alpha_{3}}, \quad e^{\phi} \simeq \phi_{0} r^{\alpha_{4}}
$$

and $Y \simeq y_{0} e^{\alpha \phi} \simeq y_{0} \phi_{0}^{\alpha} r^{\alpha \alpha_{4}}$. We will not explicitly solve the related Schrodinger equations, however, by analysing the behavior of Schrodinger potentials close to IR, one can conclude about which form of the leading order of geometry should one consider if the dual phase is in an insulating phase or a semimetal phase.

The fluctuation equation (3.2) can be rewritten as $\left(\frac{u^{2} Y}{\sqrt{h}} v_{z}^{\prime}\right)^{\prime}+\frac{Y}{\sqrt{h}} \omega^{2} v_{z}=0$. Introducing $\xi$ and $\tilde{v}_{z}$ as

$$
\frac{d \xi}{d r}=\frac{1}{u}, \quad \tilde{v}_{z}=C_{1} v_{z}, \quad C_{1}=\sqrt{\frac{u Y}{\sqrt{h}}}
$$

\footnotetext{
${ }^{10}$ Obviously for the model considered in the main text, the IR geometries found in section 2.1 are of this form.
} 
we write the equation (3.2) as a Schrödinger equation

$$
-\frac{d^{2} \tilde{v}_{z}}{d \xi^{2}}+V_{\mathrm{eff}}(\xi) \tilde{v}_{z}=\omega^{2} \tilde{v}_{z}
$$

with the Schrödinger potential

$$
V_{\text {eff }}=\frac{\left(u Y h^{-1 / 2}\right)^{\prime}}{4}\left(\frac{u}{Y h^{-1 / 2}}\right)^{\prime}+\frac{u^{2}\left(u Y h^{-1 / 2}\right)^{\prime \prime}}{2 u Y h^{-1 / 2}}
$$

where the prime is the derivative with respect to $r$. One can transfer it back to the coordinate $\xi$ using (C.2).

Depending on the values of $\alpha_{1}$, in the new radial variable of form (C.2), the horizon is located either at $\xi=-\infty$ (for $\left.\alpha_{1} \geq 1\right)$ or $\xi=c$ (for $\left.\alpha_{1}<1\right)$. Near the UV boundary $\xi \rightarrow$ $0^{-}$, we have a divergent potential $V_{\text {eff }} \propto(-\xi)^{-2}$. Near the horizon, we have $V_{\text {eff }} \propto r^{2 \alpha_{1}-2}$ and its behavior will depend on $\alpha_{1}$.

- When $\alpha_{1}=1$, the effective potential is a constant at IR, with $V_{\mathrm{IR}}=\frac{u_{0}^{2}}{4}\left(1+\alpha \alpha_{4}-\frac{\alpha_{2}}{2}\right)^{2}$. When this constant value is positive, the system is in the phase with a hard gap. One can check that in the model studied in the main text $(2.11) V_{\mathrm{IR}}=\frac{1}{16}$, thus we have a hard gap with width $\Delta=\frac{1}{4}$.

- When $\alpha_{1}>1$, the effective potential goes to zero at IR and we have a semimetal phase.

- When $\alpha_{1}<1$, the effective potential diverges and we will have a discrete spectrum for the conductivity.

We can perform a similar analysis for the fluctuation equations (3.10), (3.11). Define $\omega_{ \pm}=\omega \pm 4 \alpha \frac{u A_{z}^{\prime}}{Y \sqrt{h}}$ we have $\left(u \sqrt{h} Y v_{ \pm}^{\prime}\right)^{\prime}+\frac{\sqrt{h} Y}{u} \omega_{ \pm}^{2} v_{ \pm}-\frac{16 u \alpha^{2} A_{z}^{\prime 2}}{\sqrt{h} Y} v_{ \pm}=0$. We make the coordinate change and redefine the variable

$$
\frac{d \xi}{d r}=\frac{1}{u}, \quad \tilde{v}_{ \pm}=C_{2} v_{ \pm}, \quad C_{2}=\sqrt{Y h^{1 / 2}}
$$

the equations (3.10), (3.11) can be written as Schrödinger equations

$$
-\frac{d^{2} \tilde{v}_{ \pm}}{d \xi^{2}}+V_{\mathrm{eff}}(\xi) \tilde{v}_{z}=\omega_{ \pm}^{2} \tilde{v}_{ \pm}
$$

with Schrödinger potential

$$
V_{\mathrm{eff}}=\frac{u}{C_{2}}\left(u C_{2}^{\prime}\right)^{\prime}+\frac{16 \alpha^{2} u^{2} A_{z}^{\prime 2}}{C_{2}^{2}}=\frac{\left(Y h^{1 / 2}\right)^{\prime}}{4}\left(\frac{u^{2}}{Y h^{1 / 2}}\right)^{\prime}+u^{2}\left[\frac{\left(Y h^{1 / 2}\right)^{\prime \prime}}{2 Y h^{1 / 2}}+\frac{16 \alpha^{2} A_{z}^{\prime 2}}{Y^{2} h}\right] .
$$

The location of the horizon in the coordinate $\xi$ is the same as in the previous case, which depends on the value of $\alpha_{1}$ in (C.1). Close to the UV boundary $\xi \rightarrow 0^{-}, V_{\text {eff }} \propto(-\xi)^{-2}$. When $\alpha_{1}=1$, we have $V_{\mathrm{IR}}=\frac{1}{16}$ and from (3.13) we know that there is a hard gap in the transport $\operatorname{Re}\left[\sigma_{T}\right]$. 
Open Access. This article is distributed under the terms of the Creative Commons Attribution License (CC-BY 4.0), which permits any use, distribution and reproduction in any medium, provided the original author(s) and source are credited.

\section{References}

[1] E. Witten, Three lectures on topological phases of matter, Riv. Nuovo Cim. 39 (2016) 313 [arXiv: 1510.07698] [INSPIRE].

[2] N.P. Armitage, E.J. Mele and A. Vishwanath, Weyl and Dirac semimetals in three dimensional solids, Rev. Mod. Phys. 90 (2018) 015001 [arXiv: 1705.01111] [InSPIRE].

[3] A.A. Burkov, M.D. Hook and L. Balents, Topological nodal semimetals, Phys. Rev. B 84 (2011) 235126 [arXiv:1110.1089].

[4] K. Landsteiner, Notes on anomaly induced transport, Acta Phys. Polon. B 47 (2016) 2617 [arXiv: 1610.04413] [INSPIRE].

[5] J. Zaanen, Electrons go with the flow in exotic material systems, Science 351 (2016) 1026.

[6] J. Zaanen, Y.W. Sun, Y. Liu and K. Schalm, Holographic duality in condensed matter physics, Cambridge University Press, Cambridge U.K. (2015).

[7] M. Ammon and J. Erdmenger, Gauge/gravity duality: foundations and applications, Cambridge University Press, Cambridge U.K. (2015).

[8] S.A. Hartnoll, A. Lucas and S. Sachdev, Holographic quantum matter, arXiv:1612.07324 [INSPIRE].

[9] K. Landsteiner, Y. Liu and Y.-W. Sun, Quantum phase transition between a topological and a trivial semimetal from holography, Phys. Rev. Lett. 116 (2016) 081602 [arXiv:1511.05505] [INSPIRE].

[10] K. Landsteiner and Y. Liu, The holographic Weyl semi-metal, Phys. Lett. B 753 (2016) 453 [arXiv:1505.04772] [INSPIRE].

[11] U. Gürsoy et al., Holographic models for undoped Weyl semimetals, JHEP 04 (2013) 127 [arXiv: 1209.2593] [INSPIRE].

[12] K. Hashimoto, S. Kinoshita, K. Murata and T. Oka, Holographic Floquet states I: a strongly coupled Weyl semimetal, JHEP 05 (2017) 127 [arXiv:1611.03702] [INSPIRE].

[13] M. Ammon, M. Heinrich, A. Jiménez-Alba and S. Moeckel, Surface states in holographic Weyl semimetals, Phys. Rev. Lett. 118 (2017) 201601 [arXiv: 1612.00836] [INSPIRE].

[14] Y. Liu and Y.-W. Sun, Topological invariants for holographic semimetals, JHEP 10 (2018) 189 [arXiv: 1809. 00513] [INSPIRE].

[15] Y. Liu and Y.-W. Sun, Topological nodal line semimetals in holography, JHEP 12 (2018) 072 [arXiv: 1801.09357] [INSPIRE].

[16] K. Landsteiner, Y. Liu and Y.-W. Sun, Odd viscosity in the quantum critical region of a holographic Weyl semimetal, Phys. Rev. Lett. 117 (2016) 081604 [arXiv:1604.01346] [INSPIRE].

[17] G. Grignani, A. Marini, F. Pena-Benitez and S. Speziali, AC conductivity for a holographic Weyl Semimetal, JHEP 03 (2017) 125 [arXiv: 1612.00486] [INSPIRE]. 
[18] C. Copetti, J. Fernández-Pendás and K. Landsteiner, Axial Hall effect and universality of holographic Weyl semi-metals, JHEP 02 (2017) 138 [arXiv:1611.08125] [INSPIRE].

[19] M. Ammon, M. Baggioli, A. Jiménez-Alba and S. Moeckel, A smeared quantum phase transition in disordered holography, JHEP 04 (2018) 068 [arXiv:1802.08650] [INSPIRE].

[20] M. Baggioli, B. Padhi, P.W. Phillips and C. Setty, Conjecture on the butterfly velocity across a quantum phase transition, JHEP 07 (2018) 049 [arXiv: 1805.01470] [INSPIRE].

[21] A.A. Burkov and L. Balents, Weyl semimetal in a topological insulator multilayer, Phys. Rev. Lett. 107 (2011) 127205 [arXiv: 1105.5138] [INSPIRE].

[22] B. Roy, P. Goswami and V. Juricic, Interacting Weyl fermions: phases, phase transitions and global phase diagram, Phys. Rev. B 95 (2017) 201102 [arXiv:1610.05762] [INSPIRE].

[23] C.Z. Chen et al., Disorder and metal-insulator transitions in Weyl semimetals, Phys. Rev. Lett. 115 (2015) 246603 [arXiv: 1507.00128].

[24] B. Roy, R.-J. Slager and V. Juricic, Global phase diagram of a dirty Weyl liquid and emergent superuniversality, Phys. Rev. X 8 (2018) 031076 [arXiv:1610.08973] [INSPIRE].

[25] M. Ammon et al., On holographic p-wave superfluids with back-reaction, Phys. Lett. B 686 (2010) 192 [arXiv:0912.3515] [INSPIRE].

[26] E. Kiritsis and J. Ren, On holographic insulators and supersolids, JHEP 09 (2015) 168 [arXiv: 1503.03481] [INSPIRE].

[27] S.S. Gubser, Curvature singularities: the Good, the bad and the naked, Adv. Theor. Math. Phys. 4 (2000) 679 [hep-th/0002160] [INSPIRE].

[28] C. Charmousis et al., Effective Holographic Theories for low-temperature condensed matter systems, JHEP 11 (2010) 151 [arXiv:1005.4690] [INSPIRE].

[29] L. Girardello, M. Petrini, M. Porrati and A. Zaffaroni, Confinement and condensates without fine tuning in supergravity duals of gauge theories, JHEP 05 (1999) 026 [hep-th/9903026] [INSPIRE].

[30] H. Liu and M. Mezei, Probing renormalization group flows using entanglement entropy, JHEP 01 (2014) 098 [arXiv: 1309.6935] [INSPIRE].

[31] M. Bianchi, D.Z. Freedman and K. Skenderis, How to go with an RG flow, JHEP 08 (2001) 041 [hep-th/0105276] [INSPIRE].

[32] G.T. Horowitz and M.M. Roberts, Zero temperature limit of holographic superconductors, JHEP 11 (2009) 015 [arXiv:0908.3677] [INSPIRE].

[33] S.S. Gubser and A. Nellore, Ground states of holographic superconductors, Phys. Rev. D 80 (2009) 105007 [arXiv: 0908.1972] [INSPIRE].

[34] S.A. Hartnoll and L. Huijse, Fractionalization of holographic Fermi surfaces, Class. Quant. Grav. 29 (2012) 194001 [arXiv:1111.2606] [INSPIRE].

[35] A. Donos and S.A. Hartnoll, Interaction-driven localization in holography, Nature Phys. 9 (2013) 649 [arXiv: 1212.2998] [INSPIRE].

[36] A.G. Grushin, Consequences of a condensed matter realization of Lorentz violating QED in Weyl semi-metals, Phys. Rev. D 86 (2012) 045001 [arXiv:1205.3722] [InSPIRE].

[37] A.A. Zyuzin and A.A. Burkov, Topological response in Weyl semimetals and the chiral anomaly, Phys. Rev. B 86 (2012) 115133 [arXiv:1206.1868] [inSPIRE]. 
[38] P. Goswami and S. Tewari, Axionic field theory of $(3+1)$-dimensional Weyl semimetals, Phys. Rev. B 88 (2013) 245107 [arXiv: 1210.6352] [INSPIRE].

[39] Y. Yoshimura et al., Comparative study of Weyl semimetal and topological/Chern insulators: Thin-film point of view, Phys. Rev. B 94 (2016) 235414 [arXiv: 1606.02091] [INSPIRE].

[40] L. Lu and Z. Wang, Topological one-way fiber of second Chern number, arXiv:1611.01998. 